\title{
Researchon Classification Techniques in Data Mining
}

\author{
O.Bhaskaru, M.Sree Devi
}

\begin{abstract}
Data Mining means a procedure to extracting the information out of large data. Data miningapproaches includes classification, association rule, clustering, etc. Data mining is applied in four stages such as data sources, data extrapolation / gathering, modeling and deploying modules. Classification is a method in data mining to predict the group membership of data instances. It's an method useful in data mining with vast applications for classifying the different types of data used in almost every fields. Classification is giving a class label to in determine set of cases. In this survey, we would like discuss Bayesian classification, rules based classification, Decision trees \&neural network.
\end{abstract}

Keywords: Data Mining, Classification, Bayesian, Decision Trees, Neural Network.

\section{INTRODUCTION}

Data Mining is the process of analyzing data from different perspective and gaining the knowledge from large amount of large data[1]. Data mining is growing in various applications widely like analyzing the process of Controlling Production, Fraud Detection, Retention of Customer Science Exploration, and market analysis. These days there is tremendous measure of information being gathered and put away in databases wherever over the globe. The inclination is to gradual expanding a endless amount of time. It is not hard to find databases with Terabytes of data in enterprises and research centers.

\section{Classification in Data Mining:}

Classification is giving a class label in determined set of cases.

1. Supervised learning 2.Unsupervised learning Figure-1 represented the data mining classification.

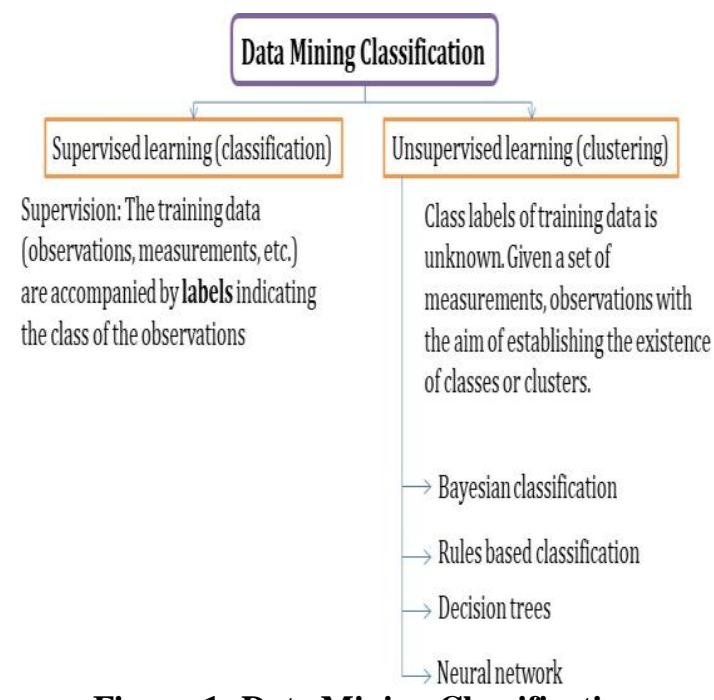

Figure-1: Data Mining Classification

\section{BAYESIANCLASSIFICATION:}

The algorithm of Naive Bayes is a probabilistic classifier that discover a set of probabilities by tallying the recurrence and mixes of qualities in a given informational collection. Bayesian arrangement depends on Bayes' Theorem.In Bayes theorem two types of probabilities such as Posterior Probability $[\mathrm{P}(\mathrm{H} / \mathrm{X})]$ and Prior Probability $[\mathrm{P}(\mathrm{H})]$. Here ' $\mathrm{X}$ ' indicates data tuple (Class) and ' $\mathrm{H}$ ' is some hypothesis. Bayes theorem was represented in figure-2

\begin{tabular}{|l|}
\hline$P(H \mid \mathbf{X})=\frac{P(\mathbf{X} \mid H) P(H)}{P(\mathbf{X})}=P(\mathbf{X} \mid H) \times P(H) / P(\mathbf{X})$ \\
- Let $\mathrm{X}$ be a data sample: class label is unknown \\
- Let $\mathrm{H}$ be a hypothesis that $\mathrm{X}$ belongs to class $\mathrm{C}$ \\
- hypsification is to determine $\mathrm{P}(\mathrm{H} \mid \mathrm{X})$, the probability that the \\
- $\mathrm{P}(\mathrm{X} \mid \mathrm{H})$ :The probability of observing the sample $\mathrm{X}$,given that the \\
hypothesis holds
\end{tabular}

Figure-2: Bayes' Theorem

\section{VPrediction based totally on Bayes' Theorem:}

Given records ' $X$ ', posteriori opportunity of speculation $X)^{\prime}$, follows the Bayes' theorem. that is regarded as "posteriori $=$ chance $\mathrm{x}$ earlier/evidence". Predicts ' $\mathrm{X}$ ' belongs to ' $\mathrm{C} i$ ' if the possibility 'percent. $\mathrm{X}$ )' is maximum in

Revised Manuscript Received on April 12, 2019.

O.Bhaskaru,Research scholar, CSE Department, KoneruLakshmaiah Education Foundation,Green Fields, Guntur District, Vaddeswaram,Andhra Pradesh 522502, India.(bha_jesus@yahoo.co.in.)

Dr.M.Sree Devi,Professor, CSE Department,KoneruLakshmaiah Education Foundation,Green Fields, Guntur District, vaddeswaram,Andhra Pradesh 522502, India (msreedevi_27@kluniversity.in.) 


\section{Research On Classification Techniques In Data Mining}

all of the '\%.X)' for every 'ok' commands. realistic issue is requires introductory studying of severa probabilities, inclusive of large computational fee [2].

\section{Classification is to derive the Maximum Posteriori:}

$$
P\left(C_{i} \mid \mathbf{X}\right)=\frac{P\left(\mathbf{X} \mid C_{i}\right) P\left(C_{i}\right)}{P(\mathbf{X})}
$$

\section{$\mathrm{P}(\mathrm{X})$ is constant for all classes}

$$
P\left(C_{i} \mid \mathbf{X}\right)=P\left(\mathbf{X} \mid C_{i}\right) P\left(C_{i}\right)
$$

Let $\mathrm{D}$ be a training set of tuples and their associated class labels, and each tuple is represented be an n-D attribute vector $\mathrm{X}=(\mathrm{x} 1, \mathrm{x} 2, \mathrm{x} 3)$. suppose there are $\mathrm{m}$ classes $\mathrm{C} 1, \mathrm{C} 2, \ldots . \mathrm{Cm}$.classificatin is to derive the maximum posteriori,i.e, the maximal $\mathrm{P}(\mathrm{Ci} \mid \mathrm{X})$,derived Bayes'theorem.

\section{Figure-3: Maximum Posteriori}

\section{RULES BASEDCLASSIFICATION:}

Policies are a way of representing information or bits of knowledge. A rule-based definitely classifier makes use of a fixed of 'IF

-THEN' regulations for sophistication [4-5].constitute the knowledge within the form of 'IF-THEN' hints.

R: IF (age $==$ medium) AND (computer knowledge $==$ no) THEN buys_computer=no.

conflict resolutions is needed If multiple rule are added about.

i. duration ordering: assign the very super priority to the triggering guidelines which have the toughest requirement

ii. magnificence primarily based ordering: decrementing the order of the misclassification fee / splendor[4].

iii. Rule primarily based definitely ordering : hints might be organized into the handiest precedence list, based totally on few measures of the guideline of thumb of thumb remarkable

\section{Sequential protective approach:}

i. Sequential covering set of guidelines: gain rules exactly from schooling facts.

ii. Sequential shielding algorithms: CN2, FOIL, AQ, RIPPER.

iii. Sequentially the guidelines are located out, every for the given splendor ' $\mathrm{Ci}$ ' will cover greater tuples of ' $\mathrm{Ci}$ ' but few or none of tuples of the opportunity schooling.

iv. Steps:

a. reading of regulations is finished one after the alternative.

b. on every occasion at the same time as studying the guideline, the tuples covered through the regulations are removed.

v. Repeat the device on the final tuples until the issue that give up state of affairs. vi. studying a difficult and fast of guidelines simultaneously [3].

Sequential defensive set of recommendations:

At the identical time as (enough target tuples left) generate a rule cast off effective intention tuples enjoyable this rule.

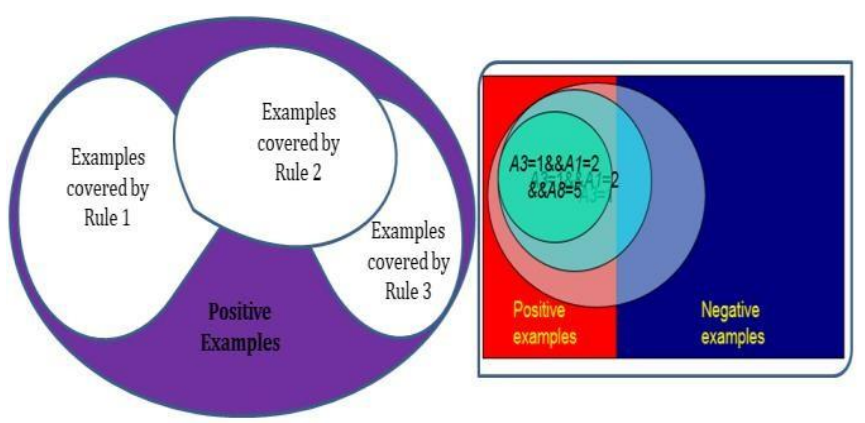

Figure-4: Positive and Negative Examples

Rule era:

Even as (real) find out the extremely good predicate ' $p$ '

iffoil_gain $(p)>$ threshold then upload ' $p$ ' to modern rule else harm.

a way to take a look at One Rule?

i. start with the most extensive rule feasible: condition = empty.

ii. Integrating new attributes with the resource of adopting a grasping depth first technique.

iii. choosing one this is maximum complements the identical vintage excellent.

Rule outstanding measures: do not forget accuracy and the coverage. Foil_gain (in Ripper and Foil): places price on info_gain via giving to state of affairs.

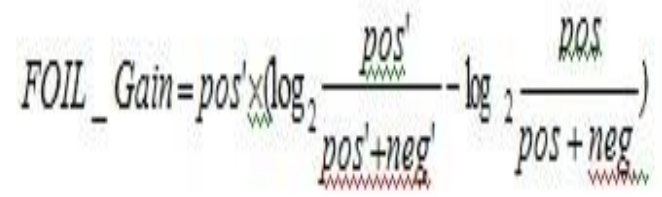

pruning the rule based upon independent set of the test tuples positive/negative. If FOIL_Prune is grater for pruned version of the $R$, pruneR.

$$
\begin{aligned}
& \text { FOIL_Prune }(R)={ }^{\text {pos }- \text { neg }} \\
& \text { pos+neg }
\end{aligned}
$$

\section{PREFERENCE TIMBER:}

Constructing choice tree: desire tree assembles or regression models as a tree shape. It divides a dataset into littler and littler subsets whilst inside the interim a associated selection tree is step by step superior [6-8].The final final consequences might be a tree with selection nodes, leaf nodes and branches. each node in a choice tree represents a feature in an example to be categorised, and every branch represents a fee that the node can expect. times are ordered starting at the root node and prepared relying on their component values. 


\section{Step approach:}

Tree manufacturing

1. Taking an feature for dividing of given records

2. Separation the given facts into sets at the concept of this function

3. For each set made above - repeat 1 st and second steps till you discover leaf nodes in each one of the branches of the tree-Terminate

\section{B. Tree Pruning (Optimization)}

Discover and eliminate branches inside the choice Tree that aren't useful for class

\section{i. Pre-Pruning:}

Halt tree introduction early don't break up a node if this will bring about the goodness degree falling beneath a threshold. difficult to definitely get keep of the right threshold.

ii. located up Pruning:

remove branches from "really grown" tree-get a sequence of pruned wooden Use the set of records incredible from training information for identifying which one is the "extremely good pruned tree".

Tree creation example:

training facts set: Buys pc

schooling information set: Buys computer

Given Data:

Table-1: Given data

\begin{tabular}{|c|c|c|c|c|}
\hline Age & Income & Student & Credit_rating & Buys_Laptop \\
\hline$<=30$ & High & No & Fair & No \\
\hline$<=30$ & High & No & Excellent & No \\
\hline $31 \ldots . .40$ & High & No & Fair & Yes \\
\hline$>40$ & Medium & No & Fair & Yes \\
\hline$>40$ & Low & Yes & Fair & Yes \\
\hline$>40$ & Low & Yes & Excellent & No \\
\hline $31 \ldots . .40$ & Low & Yes & Excellent & Yes \\
\hline$<=30$ & Medium & No & Fair & No \\
\hline$<=30$ & Low & Yes & Fair & Yes \\
\hline$>40$ & Medium & Yes & Fair & Yes \\
\hline$<=30$ & Medium & Yes & Excellent & Yes \\
\hline $31 \ldots 40$ & Medium & No & Excellent & Yes \\
\hline $31 \ldots . .40$ & High & Yes & Fair & Yes \\
\hline$>40$ & Medium & No & Excellent & No \\
\hline
\end{tabular}

Three Data Sets formed after division at root node on the point of "age" attribute.

\section{FINAL DECISION TREE:}

On the basis of tree created in the manner described, classify a test sample (age, student, credit rating, buys Laptop) (<=30, yes, excellent,?)-Will this student buy Laptop?

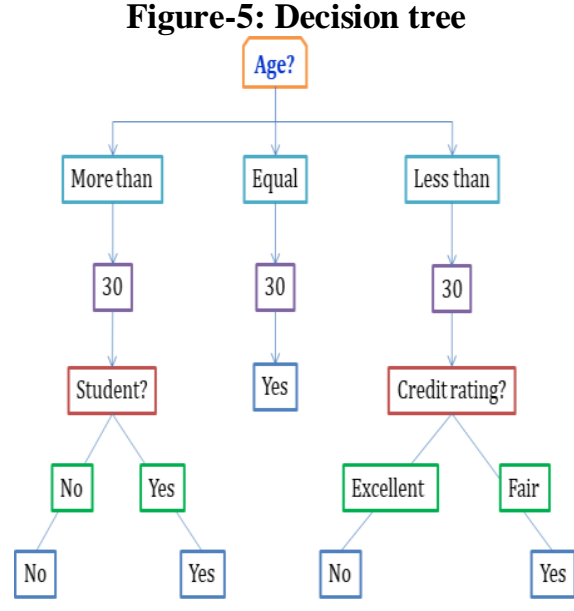

IF age $<=30$ AND student $==$ no THEN buys_Laptop $=$ 'no' IF age $<=30$ AND student $==$ yes THEN buys_Laptop $=$ 'yes'

IF age $==31 \ldots 40$ THEN buys_Laptop $=$ 'yes'

IF age $=>40$ AND credit_rating $==$ excellent THEN

buys_Laptop $=$ 'yes'

IF age $<=30$ AND credit_rating == fair THEN

buys_Laptop= 'no'

Table: Example

\section{NEURAL COMMUNITY\& RESULTS:}

It denotes a brain metaphor for the facts processing. the ones models are biologically stimulated instead of a simply best duplicate of the way the mind is surely functioning. It have been showing to be promising systems in forecasting business corporation type programs due to their functionality to "analyze" from the facts, their nonparametric nature and their capacity to generalize [9] and programs.

In most times "Neural network" is an adaptive gadget that modifies its structure supported inner or outside statistics that actions via the community within the mastering phase.

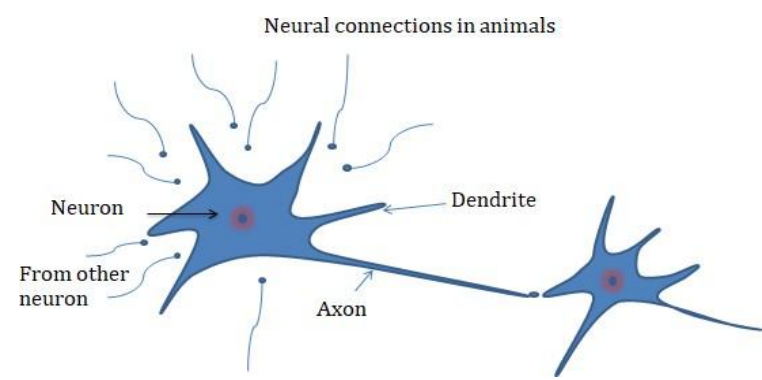

Figure 6: Neural connections and Network 


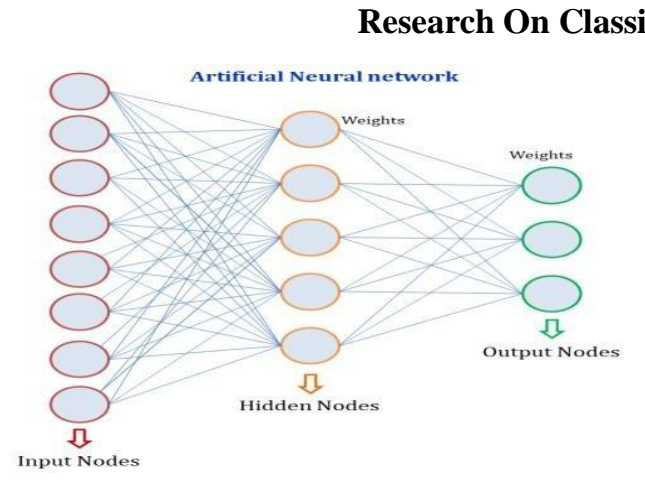

In Figure-6: neural networks are non linear statistical data modeling equipment. those neural networks used to version hard relationships amongst outputs and inputs or to are looking for for out styles among information. using neural networks as a device, information warehousing businesses have grow to be statistics from datasets in the method this is referred to as statistics mining. The difference most of the ones facts warehouses and conventional databases is that there's actual manipulation and pass- fertilization of the data helping the customers makes right sufficient informed picks [10].This technique is utilized in clustering, magnificence, function mining, pattern recognition and prediction. It imitates the neurons form of animals, bases on the Hebb studying rule and M-P version, consequently it's a matrix of allocated form. thru the schooling statistics mining, this system frequently calculates the weights the neural network related. This version is split into the 3 kinds:

a. Feed in advance networks (FFN): It regards the perception lower another time propagation version and moreover the feature network as representatives, and within the important applied within the areas like prediction and pattern recognition.

b. feedback community (FBN): It regards non-prevent version and Hopfield version that is used as an ordinary magnificence of example, and in the most essential used for optimization calculation and associative reminiscence.

c. Self agency networks (SON): it regards adaptive resonance idea version and Kohonen version as representatives and inside the primary used for cluster assessment.

\section{Feed in advance Neural community:}

One of the only feed in advance neural networks (FFNN), like in figure, consists of 3 layers.
i. input layer(IL)
ii. Hidden layer(HL)
iii. Output layer(OL)

In each layer there are one or extra processing factors (PEs). It is meant to imitate the neurons in the mind and that is why they will be frequently called as nodes or neurons. A PE gets inputs from each the preceding layer or the outside global. There are connections among the PEs in each layer that have a weight related to them. This weight is adjusted at some point of training. The records travels best inside the sooner path within the network - there aren't any remarks loops.

\section{The simplified approach for schooling a FFNN:}

1. Input information is examined to the network and it's propagated via the community until it reaches the output layer. This in advance technique offers a anticipated output.

2. This predicted output is eliminated from the real output and an mistakes price for the networks is calculated.

3. This neural community makes use of supervised analyzing, which a number of the principle instances is decrease decrease back propagation, to educate the community. decreasedecrease lower back propagation is a reading set of rules for adjusting the weights. It begins offevolvedoffevolved with the weights in some of the output layer PE's and additionally the ultimate hidden layer PE's and works backwards in the network.

4. As speedy because the decrease decrease lower lower back propagation has completed, the in advance method begins offevolvedoffevolvedoffevolved over and over, and this cycle might be a keeps the device until the mistake amongst expected and real outputs is reduced

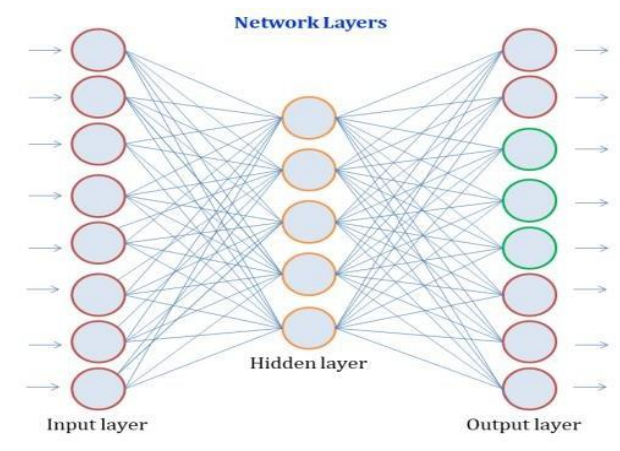

Figure-7: Layers

\section{CONCLUSION}

Bayesian community and desire trees are have absolutely unique operational profiles in which one is relatively accurate and the other isn't always and inversely. On the other, rule classifiers and desire timber have a equal operational profile. The cause of kind quit give up end result integration algorithms is to get greater particular, correct and positive machine results. unique techniques are endorsed for the making of ensemble of classifiers. regardless of the fact that numerous strategies of ensemble introduction have been proposed, till now no clean way of which technique is proper. a number of the class strategies produce high-quality prediction inside the phenotype. but, software of kind techniques to many numbers of markers has a capability danger gaining up randomly associated markers. three strategies had been carried out specifically Naive Bayes, choice timber \& Neural Networks. From the effects Neural Networks gives actual results as examine to Naive Bayes and choice wood.

\section{REFERENCES}

1. Frawley and G. Piatetsky -Shapiro, Knowledge Discovery in Databases: An Overview. Published by the AAAI Press/ The MIT Press, Menlo Park, C.A1996. 
2. Baik, S. Bala, J. (2004), A Decision Tree Algorithm for Distributed Data Mining: Towards NetworkIntrusion Detection, Lecture Notes in Computer Science, Volume 3046, Pages $206-212$.

3. Bouckaert, R. (2004), Naive Bayes Classifiers That Perform Well with Continuous Variables, Lecture Notes in Computer Science, Volume 3339, Pages 1089-1094.

4. Cheng, J. \& Greiner, R. (2001). Learning Bayesian Belief Network Classifiers: Algorithms and System, In Stroulia, E.\&Matwin, S. (ed.), AI 2001, 141-151, LNAI 2056

5. Jensen, F. (1996). An Introduction to Bayesian Networks.Springer.

6. McSherry, D. (1999). Strategic induction of decision trees. Knowledge-Based Systems, 12(5-6):269-275.

7. Bhavani,Thura-is-ingham,"Data-mining Technologies, Techniques tools \& Trends", CRCPress

8. Bradley, I., Introduction to Neural Networks, Multinet Systems Pty Ltd1997.

9. Fausett, Laurene (1994), Fundamentals of Neural Networks: Architectures, Algorithms and Applications,Prentice-Hall, New Jersey,USA.

10. Khajanchi, Amit, Artificial Neural Networks: The next intelligence. 